\title{
A Multiclass Classification Method Based on Convolutional Neural Network for Leaf Identification
}

\author{
Saniya Khan*, Juliyana N, Manjula S, Roja Y M, Akshatha M \\ Department CSE, Vidya Vikas Institute of Engineering and Technology, Mysuru, India \\ DOI: https://doi.org/10.21467/proceedings.1.59 \\ * Corresponding author email: saniyakhan471996@gmail.com
}

\begin{abstract}
The inspection of leaf categorization system is a very important subject in the field of Botany. A classifier of leaf with high accuracy will also bring a lot of fun to people's lives. However, because of the complex background of leaves, the similarity between the different species of leaves, and the differrnces among the same species of leaves, there are still some advance in the recognition of leaf images and their exposer to various diseases. Due to this complication it is often difficult to use the appropriate Pesticides. Based on Inception-v3 model of TensorFlow platform, a methodology is projected to identify the leaf and classify the type of diseases that the leaf has been affected by, using the available dataset a neural network is trained to meet the required application and detect the type of disease and displays the possible medicines.
\end{abstract}

Index Terms- Keywords-Leaf classification; TensorFlow; inception-v3; Convolutional neural network; training the model.

\section{INTRODUCTION}

The traditional leafcategorization is mainly based on the three features: colour, shape and texture, this categorization requires people to select features for classification, and the accuracy is not very high. Convolutional neural network $(\mathrm{CNN})$ is used as an efficient recognition method which has been refined in recent years. This network avoids the complex preprocessing of the image, and people can input the original image directly based on Inceptionv3 model ofTensorFlow platform, the substitution learning technology to retrain the leaf devision datasets, which can greatly improve the accuracy of flowercategorization.

The proposed approach of CNN consists of three phases that are pre-processing, feature extraction, and classification phases. Preprocessing is a crucial step as the output is dependent on pixel data that is feed as input, later removal of unwanted pixel information (i, e., noise, background). Only the leaf is needed so the background is cropped (Sand or it could be anything based on how picture is taken) using HSV color space only the Green Component is extracted and rest of the colors are ignored. In convolutional neural network, feature extraction is done by the filter or kernel in convolutional layer, then at the end of network classification is done (based on feature extraction in all previous layers) by softmax classifier.

(C) 2018 Copyright held by the author(s). Published by AIJR Publisher in Proceedings of the $3^{\text {rd }}$ National Conference on Image Processing, Computing, Communication, Networking and Data Analytics (NCICCNDA 2018), April 28, 2018.

This is an open access article under Creative Commons Attribution-NonCommercial 4.0 International (CC BY-NC 4.0) license, which permits any non-commercial use, distribution, adaptation, and reproduction in any medium, as long as the

AijR license, which permits any non-commercial use, distribution, 
A Multiclass Classification Method Based on Convolutional Neural Network for Leaf Identification

\section{LITERATURE SURVEY}

In these days, Botany field aresupposing many benefits like fraud detection of leaf infection, availability of medicines facilities to leaves at inexpensive prices, identification of smarter treatment methodologies, construction of effective medication resource management, better customer relation, improved leaf care and infection control. Disease detection is also one of the significant areas of research in Botany. Martin Abadi, AshishAgarwal, AbhineetSaxena [et, al 2016] Nowadays, Botany field are providing several benefits like fraud detection of leaf infection, availability of medicines facilities to leaves at inexpensive prices, identification of smarter treatment methodologies, construction of effective medication resource management, better customer relation, improved leaf care and infection control. Disease detection is also one of the significant areas of research in Botany.

Accuracy $(\%)$

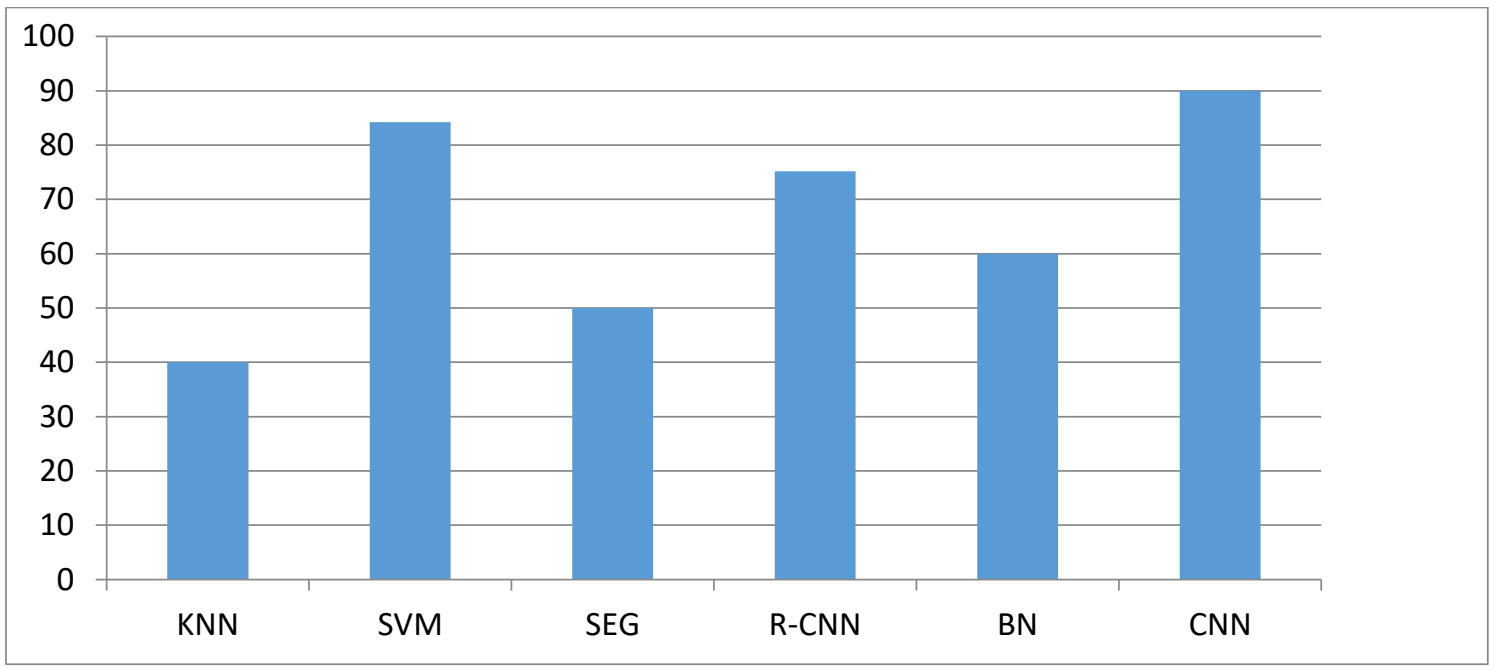

Figure:1.1 classification techniques used for convolutional neural network for leaf identification

CNN-based method provided improvements in classification accuracy over conventional method. SVM is a discriminative classifier which optimizes a separatinghyperplane by maximizing the distance between the training data. CNN is an effective classifier based on deep network learning. ANN is a classifier, inspired by a biological brain's axon, with the ability to detect patterns in the training data set, 54 which consists of assemblies of interconnected artificial neurons that provide nonlinear decision boundaries.

In Faster R-CNN, a single CNN is used for region proposals, and classifications. Bigger size typically means a large number of parameters, which makes a large network more prone to over fitting. Batch normalization potentially helps in two ways: faster learning and higher overall accuracy. The improved method also allows you to use a higher learning rate, potentially providing another boost in speed. 
Khan et al, NCICCNDA 2018, AIJR Proceedings 1, pp.375-382, 2018

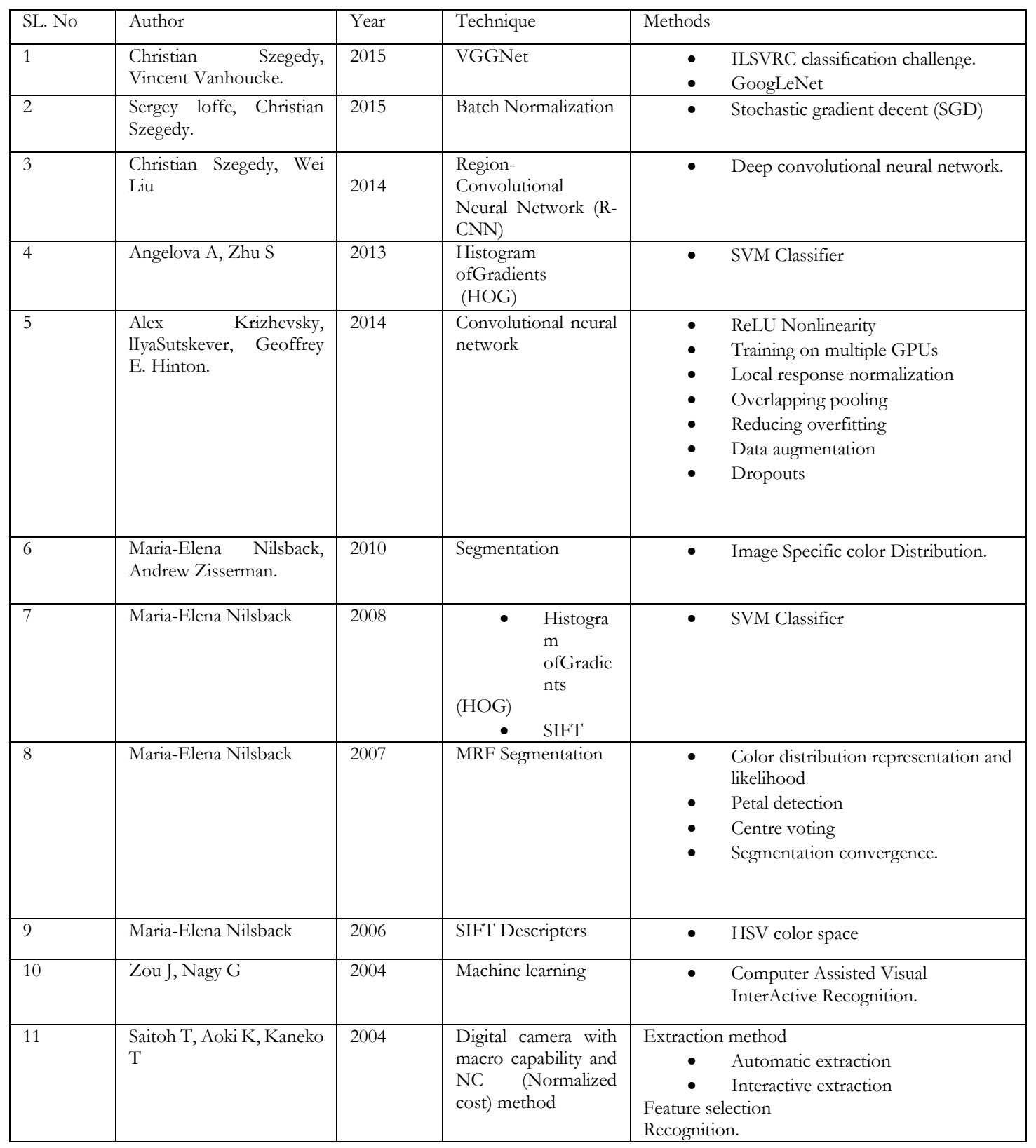

ChristianSzegedy, Wei Liu, etaI, Going Deeper with Convolutions. A deep convolutional neural network architecture codenamed Inception, which was responsible for setting the new state of the art for classification and detection in the ImageNetLargeScale Visual Recognition Challenge 2014 (ILSVRC14). The main hallmark of this architecture is the improved utilization of the computing resources inside the network. To optimize quality, the architectural decisions were based on the Hebbian principle and the intuition of multi-scale processing. Problem in

Proceedings of the $3^{\text {rd }}$ National Conference on Image Processing, Computing, Communication, Networking and Data Analytics (NCICCNDA 2018) 
A Multiclass Classification Method Based on Convolutional Neural Network for Leaf Identification

this paper is to improved utilization of the computing resources inside the network. To increase the number of layers and layer size while using dropout to address the problem of overfitting. Solution for this is, the current leading approach for object detection is the Regions with Convolutional Neural Networks (R-CNN) proposed by Girshick et al. [6]. R-CNN decomposes the overall detection problem into two sub-problems: to first utilize low-level cues such as color and super pixel consistency for potential object proposals in a category agnostic fashion, and to then use CNN classifiers to identify object categories at those locations. The most straightforward way of improving the performance of deep neural networks is by increasing their size. This includes both increasing the depth- then umber of levels-of the network and its width: the number of units at each level. Drawbacks with this is - Bigger size means large number of parameters required, the creation of high quality training sets can be tricky and expensive, and another drawback of uniformly increased network size is the dramatically increased use of computational resources.

Angelova A, Zhu S, Efficient Object Detection and Segmentation for FineGrained Recognition[C], Computer Vision and Pattern Recognition: a detection and segmentation algorithm used for the purposes of fine-grained recognition. The algorithm first detects lowlevel regions that could potentially belong to the object and then performs a full-object segmentation through propagation. Apart from segmenting the object, it can also 'zoom in' on the object, i.e. center it, normalize it for scale, and thus discount the effects of the background. Then show that combining this with a state-of-the-art classification algorithm leads to significant improvements in performance especially for datasets which are considered particularly hard for recognition. This addresses the problem of classifying objects that belong to the same basic level category, e.g. species of birds, flowers, etc. This task is often referred to as fine grained recognition and requires expert, domain specific knowledge, which very few people generally have. Therefore, developing automated recognition systems for such tasks is of much benefit to non-experts. The main challenge of fine-grained classification is undoubtedly the very fine differences between species. In this an efficient object detection and segmentation algorithm is used which is effectively used to localize the object and normalize it for scale. This method segments the possible object of interest before trying to recognize it, is much faster than previous counterparts, is applicable to a variety of different super-categories. This approach is based on identifying regions, specific of the categories of interest, at the time of detection. This propose an algorithm which combines region-based detection of the object of interest and full-object segmentation through propagation. The segmentation is applied at test time and is shown to be very useful for improving the classification performance on four challenging datasets. It tested our approach on the most contemporary and challenging datasets for fine-grained recognition improved the performances on all of them. Drawbacks of this is -Requires expert, domain specific knowledge, which very few people generally have. The main challenge of fine-grained classification is undoubtedly the very fine differences between species. A state-of-the-art 
classification algorithm leads to significant improvements in performance especially for datasets which are considered particularly hard for recognition.

Maria-Elena Nilsback, Andrew Zisserman, Delving deeper into the whorl of flower segmentation. Image Vision Computer. An algorithm for automatically segmenting flowers in color photographs. This is a challenging problem because of the sheer variety of flower classes, the variability within a class and within a particular flower, and the variability of the imaging conditions-lighting, pose, fore shortening, etc. The algorithm is evaluated on 13 flower classes and more than 750 examples. Performance is assessed against ground truth trimp segmentations. The algorithms are also compared to several previous approaches for flower segmentation. Problem in this is, the dataset has 17 flower classes, with photographs exhibiting typical variations in viewpoint, scale, illumination and background. Segmenting such photographs is challenging due to both the variety of colors and the variety of shapes. Shape also poses a challenge because of the many different types and whorls of petals. Even on the same flower there are local deformations of the petal shape. Solutions is here, they used segmentation algorithm, and introducing the flower shape model. The method is evaluated on a dataset of each class. These generalization and optimizations result in a $10 \%$ performance boost over the method of previous. This segmentation may not be perfect but is often sufficient to extract at least part of the external boundary of the flower. The generic flower shape model is then fitted to this initial segmentation in order to detect petals. The MRF segmentation is repeated using the new color models. Draw back with this is -This segmentation may not be Perfect.

Maria-Elena Nilsback, Andrew Zisserman: Automated Flower Classification over a Large Number of Classes is discussed, Investigation is done to what extent combinations of features can improve classification performance on a large dataset of similar classes. To this end introduce a 103 class flower dataset. Compute four different features for the flowers, each describing different aspects, namely the local shape/texture, the shape of the boundary, the overall spatial distribution of petals, and the color. Combining the features using a multiple kernel framework with a SVM classifier. The weights for each class are learnt using the method of varma and ray, which has achieved state of the art performance on other large dataset, such as Caltech 101/256. The dataset has a similar challenge in the number of classes, as imagebased classification systems are improving the task of classifying objects in moving onto datasets with far more categories, instead of recognizing a large number of disparate categories, investigate the problem of recognition a large number of classes within one category - that of flowers. Classifying flowers poses an extra challenge over categories such as bikes, cars and cats, because of the large similarity between classes. The difficulty lies in finding suitable features to represent colour, shape, patterns etc, and also for the classifier having the capacity to learn which features or features to use. This multiple kernel learning approach is used for flower images acquired under fairly uncontrolled image situations - the images are mainly

Proceedings of the $3^{\text {rd }}$ National Conference on Image Processing, Computing, Communication, Networking and Data Analytics (NCICCNDA 2018) 
A Multiclass Classification Method Based on Convolutional Neural Network for Leaf Identification

down loaded from the web and vary considerably in scale, resolution, lighting, clutter, quality, etc. The link we make is to automatically segment each image so that the flower is isolated in the image. This makes the recognition challenge somewhat similar in nature to that of Caltech $101 / 256$ - in that there is only a single (or very few) instances of the object in each image. Draw back of this is - The difficulty lies in finding suitable features to represent color, shape, patterns etc. It is also difficult for the classifier having the capacity to learn which features or features to use.

Saitoh T, Aoki K, Kaneko T, Automatic recognition of blooming flowers [C]. International Conference on Pattern Recognition, describes an automatic method for recognizing a blooming flower based on a photograph taken with a digital camera in natural scene. The problem of identifying an object against the background is known to be difficult. A photograph where the object (a blooming flower) is focused but the background is defocused is employed. For extracting a flower region, uses a new method that extracts a boundary by selecting a route with minimizing a sum of the local cost divided by the route length. Experiments were conducted for 600 pictures (20 pictures each for 30 species). A successful boundary extraction rate of $97 \%$ and a flower recognition rate of $90 \%$ were obtained. In order to deal with flowers with complex boundaries, a new route search method is proposed called the normalized cost (NC) method, which searches a boundary with minimizing the normalized cost i.e. the sum of local costs divided by the route length. With this NC method, entry of one point on the boundary is all the required. Feature selection consists of shape feature and color feature. Drawback with this is -Digital camera is not affordable by everyone.

\section{Existing System}

In previous studies, $\mathrm{K}$ nearest neighbors algorithm was used where classifying unknown records are relatively expensive and it requires distance computation of k-nearest neighbors. Another algorithm is SVM where the biggest limitation of the support vector approach lies in choice of the kernel and second limitation is speed and size, both in training and testing. Segmentation algorithm was used where external fragmentation is difficult and it's a costly memory management algorithm. One more algorithm is batch normalization where its difficult to estimate mean and standard deviation of input during testing and computational overhead during training.

\begin{tabular}{|c|cl|}
\hline Classification Techniques & \multicolumn{2}{|l|}{ Disadvantage of Existing System } \\
\hline $\begin{array}{c}\text { KNN } \\
\text { (K-Nearest Neighbour) }\end{array}$ & $\bullet \quad \begin{array}{l}\text { Selection of nearest matching features } \\
\text { Accuracy is low }\end{array}$ \\
\hline $\begin{array}{c}\text { SVM } \\
\text { (Support Vector Machine) }\end{array}$ & $\cdots$ & Difficult to choose kernel function parameter. \\
\hline PCA & & \\
\hline (Principal component analysis) & & \\
& & \\
\hline
\end{tabular}

ISBN: 978-81-936820-0-5 


\section{Proposed work}

Modern machine learning technique i.e. Convolutional Neural Network(Model) wherein we train the model in such a way that we get highly accurate results. This trained model is used to classify new input images.

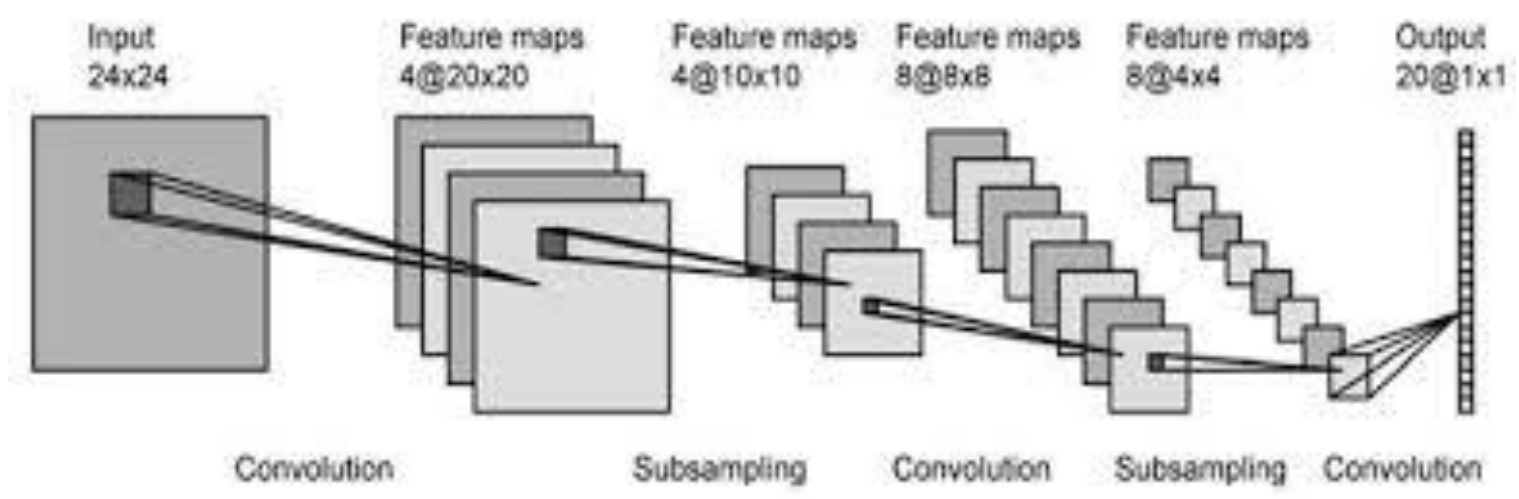

Figure 8: Proposed system Architecture

Image is passed through a series of convolutional, nonlinear, pooling (downsampling), and fully connected layers, and get an output. The output can be a single class or a probability of classes that best describes the image. The proposed approach of CNN consists of three phases that are pre-processing, feature extraction, and classification phases. Preprocessing is a crucial step as the output is dependent on pixel data that is feed as input, later removal of unwanted pixel information (i, e., noise, background). Only the leaf is needed so the background is cropped (Sand or it could be anything based on how picture is taken) using HSV color space only the Green Component is extracted and rest of the colors are ignored. In convolutional neural network, feature extraction is done by the filter or kernel in convolutional layer, then at the end of network classification is done (based on feature extraction in all previous layers) by softmax classifier.

\section{CONCLUSIONS}

During the testing phase of species of leaves, there is major increase in the accuracy of the leaf classification data. In defiance of the change in background and slight variation in the lighting condition the trained model can accurately differentiate different type of species of leaves, it's related disease and its respective possibility of cure.

\section{REFERENCES}

[1] Martin Abadi, AshishAgarwal, etaI, TensorFlow: Large-Scale Machine Learning on Heterogeneous Distributed Systems. CoRR abs/1603.04467, 2016.

[2] AbhineetSaxena, Convolutional neural networks: an illustration in TensorFlow. ACM Crossroads 22(4): 56-58, 2016.

[3] Christian Szegedy, Vincent Vanhoucke, etaI, Rethinking the Inception Architecture for Computer Vision.

Proceedings of the $3^{\text {rd }}$ National Conference on Image Processing, Computing, Communication, Networking and Data Analytics (NCICCNDA 2018) 
A Multiclass Classification Method Based on Convolutional Neural Network for Leaf Identification arXiv:1512.00567, 2015.

[4] Sergey loffe, Christian Szegedy, et al. Batch Normalization: Accelerating Deep Network Training by Reducing Internal CovarIate Shift. ICML2015: 448-456, 2015.

[5] Christian Szegedy, Wei Liu, etaI, Going Deeper with Convolutions. arXiv:1409.4842,2014.

[6] XiaodongXie, Research on Fine-Grained Classification for Visual Flower Image[D]. Xiamen University, 2014.

[7] Angelova A, Zhu S, Efficient Object Detection and Segmentation for Fine-Grained Recognition[C]. Computer Vision and Pattern Recognition: 811-818, IEEE, 2013.

[8] Alex Krizhevsky, IIyaSutskever, Geoffrey E. Hinton, ImageNet Classification with Deep Convolutional Neural Networks. NIPS 2012: 1106-1114,2014.

[9] Maria-Elena Nilsback, Andrew Zisserman, Delving deeper into the whorl of flower segmentation. Image Vision Com put. 28(6): 10491062,2010

[10] Nilsback M-E An Automatic Visual Flora -- Segmentation and Classific tiono'fFlowers Images. PhD thesis, 2009.

[11] Maria-Elena Nilsback, Andrew Zisserman: Automated Flower Classification over a Large Number of Classes. ICVGIP 2008: 722729,2008.

[12] Maria-Elena Nilsback, Andrew Zisserman: Delving into the Whorl of Flower Segmentation. BMVC 2007: 1-10,2007.

[13] Maria-Elena Nilsback, Andrew Zisserman: A Visual Vocabulary for Flower Classification. CVPR (2) 2006: $1447-$ 1454,2006 .

[14] Zou J, Nagy G, Evaluation of model-based interactive flower recognition[C]. International Conference on Pattern Recognition: 311-314, IEEE, 2004.

[15] Saitoh T, Aoki K, Kaneko T, Automatic recognition of blooming flowers[C]. International Conference on Pattern Recognition: 27-30, IEEE, 2004.

[16]https://www.google.co.in/search?q=relu+activation\&rlz=1C1CHBD_enIN758IN758\&source=lnms\&tbm=isch\&sa=X \&ved=0ahUKEwig-p2E9fzYAhWHrY8KHV2PAaUQ_AUICigB\&biw=1242\&bih=602\#imgrc=3RA1000keMvcLM:

[17]http://playground.tensorflow.org [for simple neural network demo online]

[18]https://adeshpande3.github.io/adeshpande3.github.io/A-Beginner\%27s-Guide-To-Understanding-ConvolutionalNeural-Networks/ [Simple Explanation for Neural Networks, terminologies ]

[19]https://www.youtube.com/watch?v=aircAruvnKk [visualization of neural network, watch part 2 as well for backprogpogarion]

[20]https://i.stack.imgur.com/t0zit.png 